\title{
Cerebral Venous Sinus Thrombosis Associated with Dutasteride Use
}

\author{
Bo Kyu Choi, Kyeongyeol Cheon, Bang-Hoon Cho, Jae Wook Jung, and Kyung-Yul Lee \\ Department of Neurology, Gangnam Severance Hospital, Severance Institute for Vascular and Metabolic Research, \\ Yonsei University College of Medicine, Seoul, Korea.
}

Cerebral venous thrombosis (CVT) is an uncommon cause of stroke that mainly affects young adults with known risk factors of prothrombotic conditions, pregnancy, infection, malignancy, and drugs. Dutasteride is a $5 \alpha$-reductase inhibitor that is used for benign prostate hypertrophy and androgenetic alopecia. To date, CVT caused by dutasteride use has not been reported. A 25-yearold male presented with headache and diplopia. He had taken $0.5 \mathrm{mg}$ of dutasteride every other day for 9 months to treat alopecia. A headache developed 7 months after he started taking medication, and horizontal diplopia occurred 1 month after the onset of headache. Fundus examination showed bilateral papilledema. Brain magnetic resonance imaging showed thrombosis in the left sigmoid and transverse sinuses. Headache and diplopia improved after discontinuing dutasteride and starting anticoagulation. The results from this case report indicated dutasteride as a potential cause of CVT. Presumably, the increased estrogen level due to dutasteride use caused the formation of a thrombus.

Key Words: Alopecia, venous thrombosis, 5-alpha reductase inhibitors

\section{INTRODUCTION}

Cerebral venous thrombosis (CVT) is less frequent than other types of strokes and has a different clinical presentation and etiologies.

Numerous conditions are known to cause CVT, such as prothrombotic conditions, infection, inflammatory disease, hematologic disease, malignancy, pregnancy, puerperium state, and drugs. ${ }^{1}$ Therefore, thorough etiologic investigations are needed to determine the cause of CVT. However, its underlying risk factors are not found in approximately $13 \%$ of adult patients with $\mathrm{CVT}^{2}$ Finasteride, a $5 \alpha$-reductase inhibitor, is used to treat benign prostate hypertrophy as well as androgenetic alopecia,

Received: February 28, 2020 Revised: April 23, 2020

Accepted: April 24, 2020

Corresponding author: Kyung-Yul Lee, MD, PhD, Department of Neurology, Gangnam Severance Hospital, Yonsei University College of Medicine, 211 Eonju-ro, Gangnam-gu, Seoul 06273, Korea.

Tel: 82-2-2019-3325, Fax: 82-2-3462-5904, E-mail: kylee@yuhs.ac

-The authors have no potential conflicts of interest to disclose.

(c) Copyright: Yonsei University College of Medicine 2020

This is an Open Access article distributed under the terms of the Creative Commons Attribution Non-Commercial License (https://creativecommons.org/licenses/ by-nc/4.0) which permits unrestricted non-commercial use, distribution, and reproduction in any medium, provided the original work is properly cited. and it has been reported to induce CVT as a complication. ${ }^{3}$ Dutasteride is a new $5 \alpha$-reductase inhibitor with the same indication as finasteride. Herein, we report a case of CVT caused by dutasteride use. Informed consent was obtained from the patient.

\section{CASE REPORT}

A 26-year-old male was admitted to the neurology department due to headache and horizontal diplopia. He had no previous illness or trauma history and was non-smoker. The patient had been taking $0.5 \mathrm{mg}$ of dutasteride every other day for 9 months to treat alopecia. He did not take any medication except dutasteride. A headache developed 7 months after he began taking the medication, and horizontal diplopia occurred 1 month after the onset of headache. Pulsatile headache with dizziness initially started from the occipital area and progressed to the bi-frontal and temporal areas. The patient complained of horizontal diplopia when he looked laterally to either side. Neurologic examination showed no weakness, ataxia, sensory disturbance, or visual field defect.

Initial fundus examination showed bilateral papilledema and retinal hemorrhage (Fig. 1A). To determine the cause of intra- 

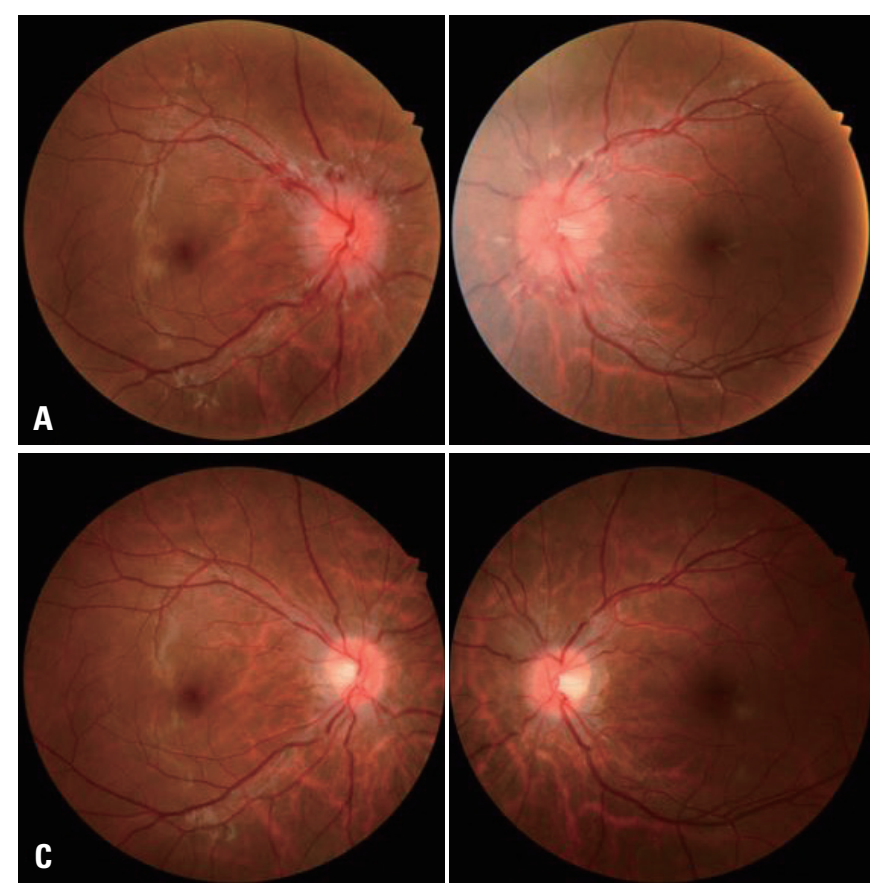
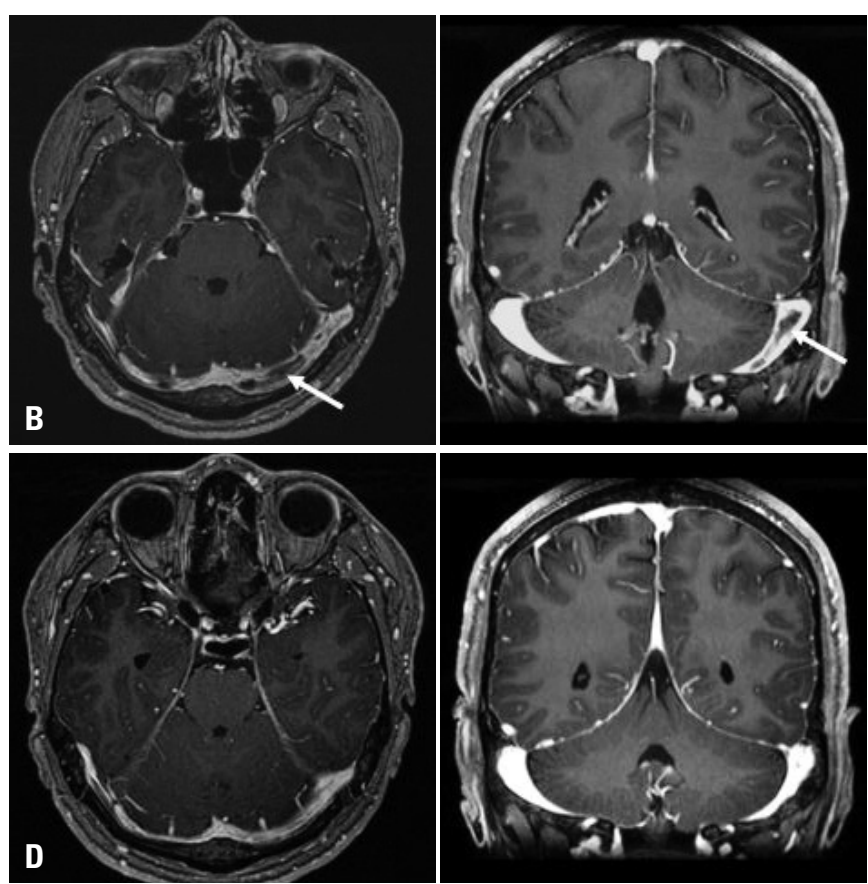

Fig. 1. (A) Initial fundus photograph shows bilateral papilledema and retinal hemorrhage. (B) Initial brain magnetic resonance imaging (MRI) shows thrombosis (arrows) in the left jugular vein, sigmoid, and transverse sinuses. (C) The papilledema is apparently improved after 6 weeks of anticoagulation treatment. (D) Follow-up MRI performed after 9 months of anticoagulation treatment shows resolution of sinus thrombosis in the left sigmoid and transverse sinuses.

cranial hypertension, brain magnetic resonance imaging (MRI) was performed on the day of admission, and it revealed thrombosis in the left jugular vein, sigmoid, and transverse sinuses (Fig. 1B). The patient showed no fever or specific findings based on blood tests for infectious conditions, including white blood cell count, erythrocyte sedimentation rate, and C-reactive protein. In addition, autoimmune antibodies, D-dimer, fibrinogen, antithrombin III, protein C, protein S, prothrombin time, activated partial thromboplastin time, and platelet count were normal. Serum estradiol level, which was measured 4 days after the discontinuation of dutasteride, was $14.2 \mathrm{pg} / \mathrm{mL}$ (normal range for male, 11.3-43.2).

There was no specific drug history to explain cerebral venous thrombosis other than dutasteride use. Therefore, dutasteride was discontinued, and intravenous anticoagulation was started to treat CVT. In addition, mannitol was used due to the bilateral papilledema for 10 days. We also used $250 \mathrm{mg}$ of acetazolamide twice a day to alleviate intracranial hypertension for 5 months. After 1 week of intravenous anticoagulation, $7.5 \mathrm{mg}$ of warfarin was given daily. After 6 weeks of oral anticoagulation treatment, the patient's symptoms were relieved, and bilateral papilledema was improved (Fig. 1C). Follow-up brain MRI performed at 9 months after anticoagulation treatment showed resolution of sinus thrombosis in the left sigmoid and transverse sinuses (Fig. 1D). Oral anticoagulation treatment was discontinued after follow-up MRI, and the patient had no symptoms since then.

\section{DISCUSSION}

Several types of drugs can cause CVT. Oral contraceptives are known to increase the risk of sinus thrombosis due to their prothrombic effects. In addition, asparaginase, cisplatin, methotrexate, lithium, and steroids can cause CVT. ${ }^{4}$ In several case reports, finasteride was found to cause CVT. Finasteride is a competitive inhibitor of type II $5 \alpha$-reductase and an intracellular enzyme that converts the androgen testosterone into $5 \alpha$-dihydrotestosterone. As a result of this mechanism, finasteride could increase serum estrone and estradiol levels and elevate the risk of CVT. ${ }^{5,6}$

In the present case, the patient also took dutasteride, a selective inhibitor of the type I and II isoforms of $5 \alpha$-reductase, which is more widely expressed and found in the skin, liver, and kidneys. The patient did not have other prothrombotic conditions, such as infection, inflammatory disease, malignan$\mathrm{cy}$, or hematologic disease, and was not using any medications that could cause CVT, except dutasteride. Previous studies did not show consistent evidence of a significant association between dutasteride therapy and risk of cardiovascular adverse events. $^{7,8}$

In this case, symptoms including headache, dizziness, and horizontal diplopia, which indicate intracranial hypertension, occurred progressively and were relieved with anticoagulation treatment, as in the previously reported CVT cases. Although there have been several studies that reported cases of CVT caused by specific drugs, dutasteride has not yet been reported to cause CVT. The present case indicates that the risk of 
CVT should be considered when dutasteride is prescribed for alopecia, benign prostate hyperplasia, or prostate cancer. In addition, a detailed history should be taken for patients who present with headache and other neurologic symptoms.

\section{AUTHOR CONTRIBUTIONS}

Conceptualization: Kyung-Yul Lee. Data curation: Bo Kyu Choi, Jae Wook Jung, and Kyeongyeol Cheon. Investigation: Bo Kyu Choi. Methodology: Bo Kyu Choi. Supervision: Bang-Hoon Cho, and Kyung-Yul Lee. Validation: Bo Kyu Choi, Bang-Hoon Cho, and Kyung-Yul Lee. Writing_original draft: Bo Kyu Choi. Writing_review \& editing: Kyeongyeol Cheon, Bang-Hoon Cho, Jae Wook Jung, and Kyung-Yul Lee. Approval of final manuscript: all authors.

\section{ORCID iDs}

Bo Kyu Choi https://orcid.org/0000-0002-0796-4043

Kyeongyeol Cheon https://orcid.org/0000-0002-2999-3945

Bang-Hoon Cho https://orcid.org/0000-0003-1730-4345

Jae Wook Jung https://orcid.org/0000-0002-9219-8522

Kyung-Yul Lee https://orcid.org/0000-0001-5585-7739

\section{REFERENCES}

1. Saposnik G, Barinagarrementeria F, Brown RD Jr, Bushnell CD,
Cucchiara B, Cushman M, et al. Diagnosis and management of cerebral venous thrombosis: a statement for healthcare professionals from the American Heart Association/American Stroke Association. Stroke 2011;42:1158-92.

2. Ferro JM, Canhão P, Stam J, Bousser MG, Barinagarrementeria F; ISCVT Investigators. Prognosis of cerebral vein and dural sinus thrombosis: results of the International Study on Cerebral Vein and Dural Sinus Thrombosis (ISCVT). Stroke 2004;35:664-70.

3. Tsuji Y, Nakayama T, Bono K, Kitamura M, Imafuku I. Two cases of stroke associated with the use of finasteride, an approved drug for male-pattern hair loss in Japan. Rinsho Shinkeigaku 2014;54:423-8.

4. Gulati D, Strbian D, Sundararajan S. Cerebral venous thrombosis: diagnosis and management. Stroke 2014;45:e16-8.

5. Yao S, Till C, Kristal AR, Goodman PJ, Hsing AW, Tangen CM, et al. Serum estrogen levels and prostate cancer risk in the prostate cancer prevention trial: a nested case-control study. Cancer Causes Control 2011;22:1121-31.

6. Kang DW, Jeong HG, Kim HR, Park CY, Pyun JM, Kim DY, et al. Finasteride induced cerebral venous thrombosis. J Korean Neurol Assoc 2015;33:238-40.

7. Skeldon SC, Macdonald EM, Law MR, Huang A, Paterson JM, Mamdani MM, et al. The cardiovascular safety of dutasteride. J Urol 2017;197:1309-14.

8. Loke YK, Ho R, Smith M, Wong O, Sandhu M, Sage W, et al. Systematic review evaluating cardiovascular events of the 5-alpha reductase inhibitor-Dutasteride. J Clin Pharm Ther 2013;38:405-15. 